\title{
Bistability, Noise, and Information Processing in Sensory Neurons
}

\author{
A. R. Bulsara \\ NCCOSC-RDT\&E Division, Code 573, San Diego, CA 92152-5000
}

\begin{abstract}
We consider the interpretation of time series data from firing events in periodically stimulated sensory neurons. A theoretical model, representing the neurons as bistable switching elements embedded in a Gaussian noise background, is considered. The cooperative effects arising through the coupling of the noise to the modulation are examined, together with their possible implications in the features of Inter-SpikeInterval Histograms (ISIHs) that are ubiquitous in neurophysiological experimental data. Our approach provides the simplest possible interpretation of the ISIHs and has been found to reproduce the salient features of experimental ISIHs.
\end{abstract}

\section{Introduction}

Neuroscientists have known for decades that sensory information is encoded in the intervals between the action potentials or "spikes" characterizing neural firing events. Statistical analyses of experimentally obtained spike trains have shown the existence of a significant random component in the inter-spike intervals. There has been speculation, of late, that the noise may actually facilitate the transmission of sensory information; certainly there exists evidence that noise in networks of neurons can dynamically alter the properties of the membrane potential and the time constants [1]. The recent re-kindling of interest in the Stochastic Resonance phenomenon [2] has lead to speculation that such nonlinear cooperative effects may occur naturally in living systems.

Stochastic resonance is a cooperative nonlinear phenomenon wherein the signal-to-noise ratio (SNR) at the output of a noisy nonlinear dynamic system driven by a weak deterministic modulation (which we shall take to be timeperiodic), can actually be enhanced by increasing the noise. Given a bistable dynamic system, for example, information is transmitted through the system in the form of switching events between the stable states (attractors) of the potential function underlying the dynamics. Suppose a periodic signal is applied; its effect is to rock the potential, alternately raising and lowering the wells. However, should its amplitude be very low (compared to the height of the potential barrier), it will not be able to induce switching. In the presence of even small amounts of noise (assumed throughout this work to be Gaussian) there will, however, always be a finite switching probability. Since the switching probability will be greater when the system is in the 'elevated' well, which occurs when the signal is at its maximum, one realizes that the noise-induced switching events may acquire some degree of coherence with the deterministic signal. A plot of SNR vs. noise strength demonstrates a characteristic bell-shaped profile. With increasing noise, the SNR rises until, for a critical noise strength, the intra-well motion is dominated by the inter-well (or switching) dynamics at which point the SNR decreases with noise; for very large noise strengths, the switching becomes noise-dominated, the particle spends very little time in the attracting states of the potential and all coherence with the periodic signal is destroyed. For low modulation frequencies, the critical noise strength (at the maximum of the SNR curve) corresponds to an approximate matching between the modulation frequency and twice the Kramers rate (this is the characteristic well-to-well switching rate in the absence of the signal), hence the somewhat misleading characterization of this effect as a 'resonance' in the physics literature.

Stochastic resonance (as characterized by the bell-shaped curve of SNR vs. noise) has not yet been directly observed in living systems with internal noise. One of its prime ingredients, noise-induced switching, has however been demonstrated explicitly, in biological experiments $[3,4]$. In all such experiments, it is common to assemble an ensemble of firing events and fit a histogram to the refractory or reset intervals occurring between the "spikes". Such InterSpike-Interval Histograms (ISIHs) are ubiquitous 
in the neurophysiological literature and, as we shall see below, can be simply and elegantly explained by simple models of neurons as bistable dynamic switching devices subject to noise. We now summarize results obtained with such a model of the response of sensory neurons to weak periodic signals embedded in noise.

Neurons as Nonlinear Dynamic Systems: Statistical Analysis of Spike Trains

Recent work by Longtin, Bulsara and Moss (LBM) [5] has demonstrated how experimental ISIHs measured, for example, on the auditory nerve fibers of squirrel monkey [6] could be explained via a new interpretation of noise-driven bistable dynamics. They introduced a simple bistable neuron model, a two-state system controlled by a double-well potential with neural firing events corresponding to transitions over the potential barrier (whose height was set such that the deterministic stimulus alone could not cause transitions). The cell dynamics were described via a variable $x(t)$, loosely denoting the membrane potential, and evolving according to

$$
\dot{x}=f(x)+q \sin \omega t+F(t),
$$

where $f(x)$ is a flow function (expressible as the gradient of a potential $U(x))$ and $F(t)$ is noise, taken to be Gaussian, delta-correlated, with zero mean and variance $2 D$. In the bistable description, the potential was taken to be the "soft" function $U(x)=a x^{2} / 2-b \ln (\cosh x)$. The system (1) has been numerically integrated, with the residence time in each potential well (these times represent the firing and quiescent intervals in our model) assembled into a histogram (the residence times density function $P(t)$ ), which displays a sequence of peaks with a characteristic spacing. Two unique sequences of temporal measurements are possible: the first measures the residence times in only one of the states of the potential and the histogram consists of peaks located at $t=n T_{0} / 2, T_{0}$ being the period of the deterministic modulation and $n$ an odd integer. The second sequence encompasses measurements of the total time spent in both potential wells, i.e. it includes the active and reset intervals; in the presence of noise, the reset intervals are of largely stochastic duration. The histogram corresponding to this sequence consists of peaks at locations $t=n T_{0}$ where $n$ is any integer. The sequence of peaks implies a form of phase locking of the neural dynamics to the stimulus. Starting from its quiescent state, the neuron attempts to fire at the first maximum of the stimulus cycle. If it fails, it will try again at the next maximum, and so on. The latter sequence is the only one observable in an experiment; the former sequence, which corresponds to the refractory events is elegantly elucidated by the LBM theory. In addition to the peak spacing in the ISIH, most of the other substantitive features of experimental ISIHs are explainable [7] via the simple model (1):

(a). Decreasing the noise intensity (keeping all other parameters fixed) leads to more peaks in the histogram since the "skipping" referred to above becomes more likely. Conversely, increasing the noise intensity tends to concentrate most of the probability in the first few peaks of the histogram. (b). In general, the probability density of residence times is well approximated by a Gamma-like distribution of the form $\left.\left.P(T)=(T /<T\rangle^{2}\right) \exp (-T /<T\rangle\right)$, where $\langle T\rangle$ is the mean of the ISIH. It is apparent that $P(T) \rightarrow 0$ and $\exp (-T /<T>)$ in the short and long time limits, respectively. For vanishingly small stimulus amplitude $q$, the distribution tends to a Gamma, conforming to experimental observations.

(c). Increasing the stimulus amplitude leads to an increase in the heights of the lower lying peaks in the ISIH.

(d). Memory effects (even within the framework of a description based on the theory of renewal processes) frequently occur, particularly at very low driving frequencies; they manifest themselves in deviations from an exponentially decaying envelope at low residence times (the first peak in the ISIH may not be the tallest one).

(e). The mean of the ISIH yields (through its inverse) the mean firing rate.

(f). The ISIH decay rate $\lambda$ (the slope of the envelope on a semi-log scale) itself depends sensitively (exponentially) on the stimulus amplitude $q$ for constant noise strength, and (again, exponentially) on the noise strength for fixed $q$. Analog simulations of the dynamics yield an extremely good fit $[5,7]$ to experimental data; the fit can be realized by changing only one parameter (the stimulus intensity or the noise intensity). The important point to note here is that the results are almost independent of the functional form of the potential $U(x)$, depending critically on the ratio of barrier height to noise variance; this ratio determines the hopping rate between the basins of attraction in the absence of noise.

The LBM theory demonstrates that the peaks of the ISIH cannot exist in the absence of noise. In fact, one could speculate that, over a 
certain range of parameters, the noise and signal play interchangeable roles in determining the shape of the ISIH. All the stimulus and noise features are encoded in the ISIH, with the phase preference (we assume perfect phase locking to the stimulus throughout; this assumptions seems to be consistent with experimental auditory nerve data at least [6]) encoded in the peak width. The noise may be used by the neuron to encode the stimulus features in the ISIH, while preserving a fine amplitude discrimination through the exponential dependence on $\lambda$. Other researchers [8] have shown that noise linearizes the mean firing rate-vs. current characteristic in neurons, producing a large dynamic range along with ISIH variations. This dynamic range is not found in noiseless Hodgkins-Huxley type models. Identifying the mean firing rate with the inverse of the mean of the ISIH seems to lead one naturally to this encoding. Although the LBM model provides an important first step in the understanding of the (possibly pivotal) role of noise in sensory information transfer, it is far from complete. The results do not appear to depend critically on the characteristics of the potential function $U(x)$ and the fundamental question: what aspects of the data are due to the statistical properties of noisy two-state systems as opposed to real (i.e. biological) properties of cells that transcend this simple description, has not been satisfactorily answered, although an important first step in this direction is afforded by recent, more rigorous work [7].

Bistable models of the type discussed above provide one of the simplest explanations of the possible mechanism underlying the processing and coding of sensory information in the nervous system. They elucidate the sequence of reset events that follow each neural firing, and noise is seen to play a pivotal role in the production of the ISIHs. The class of models discussed above represents bistability between two fixed points; the system dynamics are underpinned by the potential function $U(x)$. Other types of bistability can also occur in neurophysiology e.g. the Fitzhugh-Nagumo model represents bistability between a fixed point and a limit cycle with the transition between these attractors accompanied by a Hopf bifurcation; this model is deriveable from the Hodgkins-Huxley equations under certain conditions. The dynamics in a generic bistable system of the form (1) displays the $\mathrm{N}$ shaped flow characteristic that is known to exist in exciteable cells [9]. Recent treatments [10] of many-body problems consisting of cells coupled to "baths" of dendrites have shown that, for certain classes of neurons, models such as (1) can be derived for a macroscopic or collective variable $x(t)$ describing the many-body system, with the coefficients $a$ and $b$ in the potential being functions of all the cell and dendritic parameters (the effect of the dendritic coupling is to modify the height of the potential barrier and the spacing of the elliptic points, thereby directly affecting the switching dynamics and the structure of the ISIH). The SNR at the output of such a manybody system can be further enhanced (over its expected value for the isolated case) by the coupling to the noisy bath. The potential function for the system becomes bimodal above a critical value of $b / a$; this transition to bimodality is mediated by the noise in the dendritic bath. Since none of the cooperative effects that we have described here will occur in a monostable potential, the critical role of background noise is evident.

\section{Discussion and Conclusion}

It seems fitting to conclude this review with a question and some speculation. Clearly the question to be answered is: "Is the neuron indeed a noisy nonlinear switching element, describeable by a bistable dynamics of the form (1) (with, say, a 'soft' potential $U(x))$ ?" Certainly, there is ample evidence that noise plays a critical role in the transmission and coding of information in the nervous system. It seems reasonable then, that there should exist a form of self-regulatory mechanism such that the internal parameters of the system (i.e. the potential parameters) can be adjusted in response to the stimulus and noise characteristics. This is important, because, for given stimulus and noise, one obtains welldefined histograms for only a small range of $U_{0}$. One may speculate further that the neuron (or the network) then uses the background noise so that its response (measured via the SNR or, equivalently, through the ISIH) is optimized. This implies that the network operates close to the maximum of the stochastic resonance curve (SNR vs. noise variance) while simultaneously obtaining other information (e.g. amplitude, frequency, and phase) about the deterministic stimulus, via the ISIH. In effect, our construction and interpretation of the ISIH (together with the remarkable ability to explain most of the features of experimentally obtained ISIHs) as a natural outcome of our modelling the neuron as a noisy bistable switching element implies that sensory neurons 
may measure the stimulus intensity by comparing it to the background noise, using the (internally adjusted) potential barrier height to mediate and optimize the measurement [F. Moss 1992; private discussions]. Our studies of collective behavior in large networks show [10] that the coupling to other elements can enhance or degrade the SNR depending on the magnitudes and signs of the coupling coefficients (i.e., the excitatory or inhibitory nature of the interactions is critical).

The Inter-Spike-Interval Histograms (obtained via a bistable description of neural firing events) are not, by themselves, necessarily an indicator of the presence of stochastic resonance as an underlying cooperative effect in neurophysiology. They are, however, a product of correlated (between the noise and deterministic modulation) switching and the SNR at the output of bistable models of the form (1) has been studied elsewhere [2,11]. Various features of these histograms can, however, lend themselves to explanations based on stochastic resonance. Perhaps the most important of these features is that the heights of successive peaks pass through a maximum as a function of the noise strength [12]. So far, attempts to quantify this "resonance" as a matching of two characteristic rates have been inconclusive, largely because of the difficulty of (numerically) producing good ISIHs with low noise. The question of defining a "SNR" directly from the ISIHs is also largely unanswered. Although the bistable model (1) is striking for its simplicity, it can be criticized for lacking neurophysiological rigor. Indeed, ISIHs obtained from more conventional "Integrate-andFire" models [13] display many of the same features; however, their interpretation must be based on other considerations since stochastic resonance is not observed in such models.

Experimental investigations into the occurrence of SR in living systems are now underway. Douglass et. al. [4] have measured the SNR vs. noise strength curves in the crayfish mechanoreceptor. With externally applied noise, the SNR displays the characteristic bell-shaped response as a function of noise strength. For the internal noise case, the results are not as clear. The SNR increases monotonically as a function of the temperature of the saline bath with the temperature being used as a control over the internal noise, the crayfish having been acclimated for many weeks in high temperature environments $\left(28-35^{\circ} \mathrm{C}\right)$, but does not decrease with increasing internal noise. This result is significant in its own right; it points to the existence of a fundamental nonlinear dynamic mechanism underlying the cell response and the dynamics underlying stochastic resonance seem to be the most likely to provide explanations for the observed effects. These (albeit somewhat preliminary) results lend credence to our speculations regarding the positive role of noise in the detection and quantification of signals by sensory neurons.

\section{References}

1. E. Kaplan and R. Barlow; Vision Res. 16,745 (1976); H. Treutlein and K. Schulten; Ber. Bunsenges Phys. Chem. 89, 710 (1985).

2. See e.g., F. Moss: "Stochastic Resonance: from the Ice Ages to the Monkey's Ear", in "Some Problems in Statistical Physics" G. Weiss ed. (SIAM, Philadelphia 1993) and references therein; F. Moss, A. Bulsara and M. Shlesinger eds., Proceedings of the NATO-ARW on Stochastic Resonance in Physics and Biology: J. Stat. Phys. 70 no. $1 / 2$ (1993).

3. D. Chialvo and A.V. Apkarian; J. Stat. Phys. 70, 375 (1993).

4. J. Douglass, L. Wilkens, E. Pantazelou and F. Moss; Nature (1993), in press.

5. A. Longtin, A. Bulsara and F. Moss; Phys. Rev. Lett. 67, 656 (1991). See also Nature 352, 469 (1991).

6. J. Rose, J. Brugge, D. Anderson and J. Hind; J. Neurophys. $\underline{30}, 769$ (1967).

7. A. Longtin, A. Bulsara, D. Pierson and F. Moss; Biol. Cyb., preprint.

8. H. Treutlein and K. Schulten; Ber Bunsenges Phys. Chem. 89, 710 (1985).

9. J. Rinzel and B. Ermentrout; in "Methods in Neuronal Modelling", C. Koch and I. Segev eds. (MIT Press, Cambridge, MA 1989); L. Abbott and T. Kepler; in "Statistical Mechanics of Neural Networks", L. Garrido ed. (Springer Verlag, Berlin 1990).

10. A. Bulsara and G. Schmera; Phys. Rev. E47, 3734 (1993); A. Bulsara, A. Maren and G. Schmera; Biol. Cyb., in press; A. Bulsara and A. Maren; in "Proceedings of the First Applachian Conference on Neurodynamics", in press.

11. A. Bulsara, E. Jacobs, T. Zhou, F. Moss and L. Kiss; J. Theor. Biol. 152, 531 (1991).

12. T. Zhou, F. Moss and P. Jung; Phys. Rev. $\underline{\text { A42, }} 3161$ (1991); A. Longtin; J. Stat. Phys. 70, 309 (1993).

13. G. Gerstein and B. Mandelbrot; Biophys. J. $\underline{4}$, 41 (1968); D. Berger, K. Pribram, H. Wild and C. Bridges; Expt. Brain Res. 80, 129 (1990). 